\section{Déficits intrinsèques des cellules satellites dans la dystrophie musculaire de Duchenne}

${ }^{1}$ Sprott center for stem cell research, Institut de recherche de l'hôpital d'Ottawa, Ottawa, ON, Canada;

${ }^{2}$ Université d'Ottawa, Ottawa, ON, Canada ;

${ }^{3}$ Centre de recherche du centre hospitalier universitaire Ste-Justine, Montréal, Québec, Canada ;

4 École de réadaptation, faculté de médecine, Université de Montréal, 7077 avenue du Parc, H3N 1 X7 Montréal, Québec, Canada.

nicolas.dumont.1@umontreal.ca

Les cellules satellites dans la dystrophie musculaire de Duchenne Le muscle squelettique possède une capacité régénérative exceptionnelle lui permettant de restaurer son intégrité et ses fonctions suite à une blessure massive, en quelques semaines seulement [1]. Cette capacité est attribuable aux cellules souches musculaires adultes appelées cellules satellites. Dans le muscle sain, ces cellules sont quiescentes. Cependant, suite à un traumatisme, elles s'activent et prolifèrent rapidement, produisant ainsi un grand nombre de cellules musculaires différenciées, ou myoblastes, qui régénèreront les fibres musculaires endommagées [2]. La dystrophie musculaire de Duchenne (DMD) est une maladie dégénérative létale caractérisée par une faiblesse musculaire progressive et irréversible, et causée par une mutation dans le gène de la dystrophine, localisé sur le chromosome $X$. Ce gène code la protéine dystrophine, une grande protéine dont la région $\mathrm{N}$-terminale se lie aux filaments d'actine et la région C-terminale au complexe dystroglycane à la membrane cellulaire. Grâce à sa structure, la dystrophine permet le maintien de l'architecture et de l'intégrité des fibres musculaires. L'absence de dystrophine dans les fibres musculaires de patients DMD entraîne consécutivement la nécrose primaire des fibres, suivie de l'infiltration chronique de cellules inflammatoires et de l'accumulation de fibrose et de tissu adipeux intramusculaire $[3](\rightarrow)$.

$\rightarrow$ Voir la Synthèse de S. Dufresne et al., $m / s$ $n^{\circ} 6-7$, juin-juillet 2016, page 591
Les patients DMD présentent donc une dégénérescence des fibres musculaires et une fonte musculaire malgré la présence des cellules satellites au sein du tissu musculaire.

Toutefois, les raisons pour lesquelles les cellules satellites ne parviennent pas à régénérer efficacement le tissu musculaire demeurent incertaines. Comme les cellules satellites sont constamment sollicitées dans les muscles dystrophiques, il a été suggéré que l'épuisement de la réserve de ces cellules, causé par le raccourcissement de leurs télomères, était la cause de la perte de la capacité régénérative [4]. Néanmoins, des études chez l'homme et la souris ont démontré que le nombre de cellules satellites dans les muscles dystrophiques est élevé, et ce, même dans les stades avancés de la maladie $[5,6]$.

\section{Rôle du complexe dystroglycane dans} les cellules satellites

Si, jusqu'à récemment, l'expression de la dystrophine et des protéines du complexe dystroglycane (DGC) était restreinte aux fibres musculaires, l'analyse par puce à ADN a révélé que les cellules satellites expriment également la plupart des composants du DGC $[7,8]$. Les cellules satellites expriment ainsi la protéine dystroglycane (Dagl) qui joue un rôle clé dans leur bon fonctionnement [9]. En effet, sa délétion spécifique uniquement dans les fibres musculaires entraîne une dégénérescence musculaire moindre que celle observée suite à sa délétion généralisée dans les fibres musculaires et les cellules satellites [9]. La glycosylation de Dagl par deux glycosyltransférases, fukutine et LARGE (like-glycosyltransferase), est également essentielle pour permettre l'interaction du DGC avec la matrice extracellulaire [10]. Là encore, la délétion spécifique de la fukutine uniquement dans les fibres musculaires entraîne une dégénérescence musculaire moins importante que sa délétion dans les progéniteurs myogéniques [10]. Enfin, les cellules satellites déficientes pour LARGE présentent une capacité proliférative diminuée [11]. Ces résultats soulignent l'importance des protéines du DGC pour la fonction des cellules satellites et suggèrent que l'incapacité des cellules satellites à régénérer les muscles dystrophiques est due à des déficits cellulaires intrinsèques.

Dans une récente étude, nous avons démontré que les cellules satellites sont intrinsèquement déficientes dans la DMD [7]. L'immunomarquage de cellules satellites murines, isolées de muscles sains en régénération ou de fibres musculaires cultivées ex vivo, a révélé que la dystrophine est exprimée dans une fraction de cellules satellites 24 à 36 heures suivant leur activation (c'est-à-dire leur sortie de l'état de quiescence). De plus, nous avons montré que la dystrophine se lie à la protéine Mark2 (microtubule affinity regulating kinase 2), un acteur important pour l'établissement de la polarité cellulaire. Le complexe dystrophine-Mark2 interagit avec un autre acteur de la polarité, la protéine Pard3 (partitioning defective 3 homo$\log )$. Cette interaction induit une distribution polarisée de Mark2 et Pard3 à des 


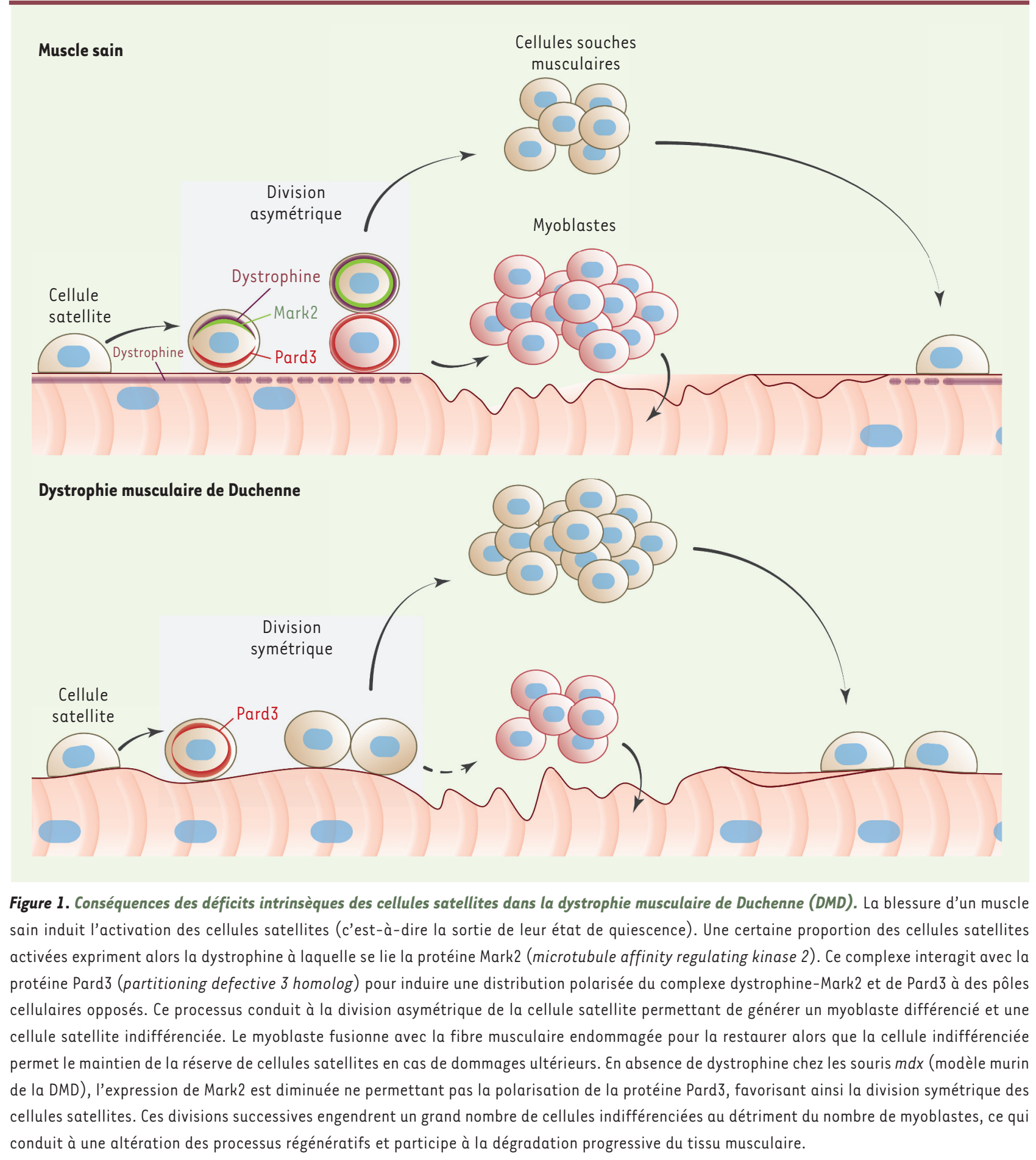
conduit à une altération des processus régénératifs et participe à la dégradation progressive du tissu musculaire.

pôles cellulaires opposés, aboutissant à une division cellulaire asymétrique (Figure 1). La division asymétrique est une caractéristique propre aux cellules souches leur permettant de générer deux cellules filles différentes. Dans le cas des cellules satellites, une cellule fille se différencie en myoblaste nécessaire à la régénération des fibres musculaires, alors que l'autre cellule fille demeure indifférenciée pour maintenir la réserve de cellules satellites.
Les cellules satellites des souris $m d x$ (modèle murin de la DMD) présentent une expression diminuée de Mark2 et un dérèglement de la polarité cellulaire [7]. Par conséquent, le nombre de divisions asymétriques des cellules satel- 
lites $m d x$ est considérablement réduit, alors que le nombre de divisions symétriques (génération de deux cellules filles indifférenciées) n'est pas affecté (Figure 1). Ceci aboutit à la réduction du nombre de myoblastes et au maintien d'un grand nombre de cellules satellites indifférenciées. Nos résultats apportent ainsi un nouvel éclairage sur les déficits intrinsèques des cellules satellites qui expliqueraient leur incapacité à régénérer adéquatement les muscles des patients DMD.

\section{Potentiel thérapeutique}

Si ces résultats nous indiquent que la DMD est une maladie plus complexe qu'anticipée, ils ouvrent également la voie à de nouvelles stratégies thérapeutiques. En effet, le développement de traitements pharmaceutiques ou génétiques visant à corriger spécifiquement les défauts intrinsèques des cellules satellites permettrait d'améliorer considérablement la fonction musculaire des patients DMD [12]. Dernièrement, il a été démontré que la surexpression de Jaggedl, un régulateur de la voie de signalisation Notch [13] $(\rightarrow)$

$(\rightarrow)$ Voir la Synthèse de A. Mayeuf et F. Relaix, $m / s n^{\circ} 5$, mai 2011, améliore le phé- page $\mathbf{5 2 1}$ notype musculaire de chiens dystrophiques [14]. Cet effet serait partiellement dû à l'augmentation de la prolifération des cellules satellites et de leur potentiel myogénique [14]. Certains inhibiteurs de voies signalétiques améliorent également la capacité régénérative des muscles de souris $m d x$. Par exemple, l'inhibition de la voie Jak2/Stat3 Uanus kinase 2 I signal transducer and activator of transcription 3) favorise la prolifération des cellules satellites de souris $m d x$ et augmente le calibre de leurs fibres musculaires [15].

Réintégrer la dystrophine dans les cellules satellites des muscles DMD pour rétablir leur capacité de division asymétrique est également une thérapie génique envisagée. Ce rétablissement permettrait d'une part de générer des myoblastes différenciés qui restaureraient l'expression de la dystrophine au sein des fibres musculaires avec lesquelles ils fusionnent et, d'autre part, d'augmenter l'efficacité à long terme du traitement en corrigeant la capacité d'auto-renouvellement des cellules satellites. Bien que la transduction d'un gène dans les cellules satellites reste un véritable défi technique, des résultats encourageants ont montré que la transduction d'un gène de micro-dystrophine dans une petite fraction de cellules satellites suffit pour maintenir son expression à long terme [16].

En somme, toutes ces études confirment l'importance des différents composants du DGC pour la fonction des cellules satellites. Bien que des travaux supplémentaires soient nécessaires pour déterminer si ces observations obtenues chez la souris sont transposables chez I'homme, ces résultats suggèrent qu'une solution thérapeutique complète devrait inclure le traitement des défauts intrinsèques des cellules satellites. $\diamond$ Cell-autonomous defects in satellite cells impair muscle regeneration in Duchenne muscular dystrophy

\section{REMERCIEMENTS}

Caroline $\varepsilon$. Brun détient une bourse post-doctorale $d u$ «Ontario Institute for Regenerative Medicine» (OIRM).

\section{LIENS D'INTÉRÊT}

Les auteurs déclarent n'avoir aucun lien d'intérêt concernant les données publiées dans cet article.

\section{RÉFÉRENCES}

1. Dumont NA, Bentzinger CF, Sincennes MC, Rudnicki MA. Satellite cells and skeletal muscle regeneration. Compr Physiol 2015 ; 5 : 1027-59.

2. Dumont NA, Wang YX, Rudnicki MA. Intrinsic and extrinsic mechanisms regulating satellite cell function. Dev Camb Engl 2015 ; 142 : 1572-81.

3. Dufresne SS, Frenette J, Dumont NA. Inflammation et régénération musculaire : une arme à double tranchant. Med Sci (Paris) 2016 ; $32: 591-7$.

4. Sacco A, Mourkioti F, Tran R, et al. Short telomeres and stem cell exhaustion model Duchenne muscular dystrophy in mdx/mTR mice. Cell 2010 ; 143 : 1059-71.

5. Jiang C, Wen $Y$, Kuroda K, et al. Notch signaling deficiency underlies age-dependent depletion of satellite cells in muscular dystrophy. Dis Model Mech $2014 ; 7$ : 997-1004.

6. Kottlors M, Kirschner J. Elevated satellite cell number in Duchenne muscular dystrophy. Cell Tissue Res $2010 ; 340: 541-8$.

7. Dumont NA, Wang $Y X$, von Maltzahn J, et al. Dystrophin expression in muscle stem cells regulates their polarity and asymmetric division. Nat Med 2015 $21: 1455-63$.

8. Fukada S, Uezumi A, Ikemoto M, et al. Molecular signature of quiescent satellite cells in adult skeletal muscle. Stem Cells Dayt Ohio 2007 ; 25 : 2448-59.

9. Cohn RD, Henry MD, Michele DE, et al. Disruption of Dagl in differentiated skeletal muscle reveals a role for dystroglycan in muscle regeneration. Cell 2002 ; $110: 639-48$

10. Kanagawa $M, Y_{u} C C$, Ito $C$, et al. Impaired viability of muscle precursor cells in muscular dystrophy with glycosylation defects and amelioration of its severe phenotype by limited gene expression. Hum Mol Genet 2013 ; 22 : 3003-15.

11. Ross J, Benn A, Jonuschies J, et al. Defects in glycosylation impair satellite stem cell function and niche composition in the muscles of the dystrophic largemyd mouse. Stem Cells 2012 ; 30 : 2330-41.

12. Dumont NA, Rudnicki MA. Targeting muscle stem cell intrinsic defects to treat Duchenne muscular dystrophy. Npj Regen Med 2016 ; 1 : 16006.

13. Mayeuf A, Relaix F. La voie Notch : du développement à la régénération du muscle squelettique. Med Sci (Paris) $2011 ; 27: 521-6$

14. Vieira NM, Elvers I, Alexander MS, et al. Jagged 1 rescues the Duchenne muscular dystrophy phenotype. Cell $2015 ; 163$ : 1204-13.

15. Tierney MT, Aydogdu T, Sala D, et al. STAT3 signaling controls satellite cell expansion and skeletal muscle repair. Nat Med 2014 ; 20 : 1182-6.

16. Kimura $\varepsilon$, Li S, Gregorevic P, et al. Dystrophin delivery to muscles of $\mathrm{mdx}$ mice using lentiviral vectors leads to myogenic progenitor targeting and stable gene expression. Mol Ther $2009 ; 18: 206-13$.

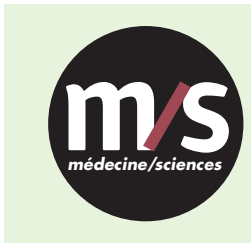

Tarifs d'abonnement $\mathrm{m} / \mathrm{s}-2016$

Abonnez-vous

à médecine/sciences
$>$ Grâce à $m / s$, vivez en direct les progrès des sciences biologiques et médicales

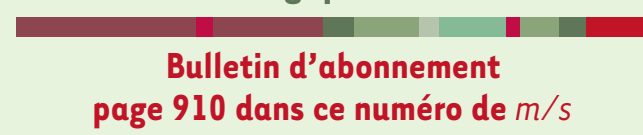

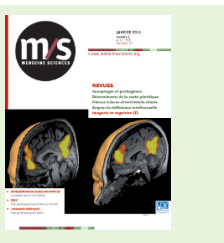

